Journal of Non-Newtonian Fluid Mechanics, 2 (1977) 169-189

(C) Elsevier Scientific Publishing Company, Amsterdam - Printed in The Netherlands

\title{
AN ELASTOHYDRODYNAMIC THEORY FOR THE RHEOLOGY OF CONCENTRATED SUSPENSIONS OF DEFORMABLE PARTICLES
}

\author{
J.D. GODDARD * \\ Department of Chemical Engineering, University of Michigan, Ann Arbor, Mich,, 48104 \\ (U.S.A.)
}

(Received July 30, 1976)

\section{Summary}

The equations which govern thin films of a Newtonian liquid confined between deformable solid surfaces are applied to the regions of near contact in a concentrated suspension of deformable particles.

For the case of slightly deformable elastic particles, one obtains the socalled "elastohydrodynamic" equations of lubrication theory.

The appropriate asymptotic solution of these equations yields estimates for the viscosity, of a form proposed earlier by Frankel and Acrivos [1] for rigid particles, as well as a relaxation time for a suspension of near spheres. The present method, which goes beyond the dissipation calculation of Frankel and Acrivos to a derivation of the full stress tensor, yields the same form of dependence of viscosity on particle concentration. However, there is an as yet unexplained difference between the methods in the value of a numerical coefficient determined by the assumed packing of the spheres.

While further work is needed on the kinetic theory for fluid suspensions, the methods employed here for the derivation of the stress tensor should have direct utility for certain solid dispersions, where it is possible to specify $a$ priori the particle-packing in the system.

\section{Introduction}

The present work is motivated by an interest in the rheological behavior of suspensions of deformable solid particles or of more general inclusions in a

* Now at the University of Southern California, Department of Chemical Engineering, University Park, Los Angeles, CA 90007. 


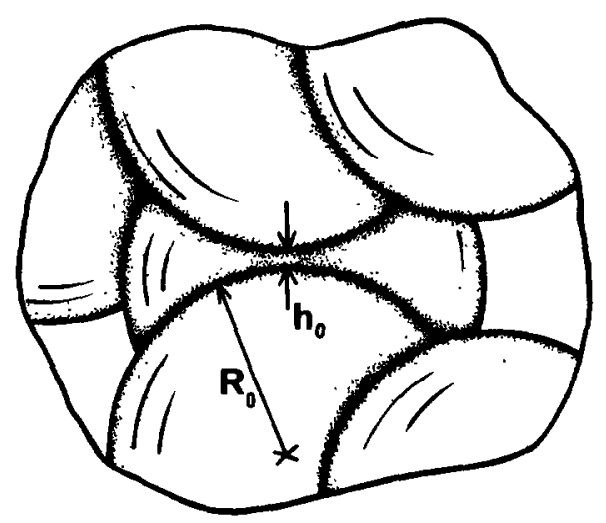

Fig. 1. Illustration of a concentrated particle suspension having $h_{0} / R_{0}<<1$.

viscous liquid. Such suspensions occur in several natural and technological settings and include such diverse materials as blood, liquid foams, and emulsions. In many cases of practical interest, the particle concentration is high and interactions between particles are strong. However, most existing theoretical treatments of the micro-rheology of dispersions are restricted to the classical dilute-suspension regime, with hydrodynamically non-interacting or weakly interacting particles, a subject which is discussed, for example, in the reviews by Brenner [2] and Batchelor [3].

The present work is concerned with the opposite extreme, that of concentrated suspensions, in the limit of nearly-touching partieles. In this limit, we shall assume that neighboring particles are physically separated from one another at points of near contact only by the intervention of a thin film of the interstitial liquid, as illustrated in Fig. 1. In addition to other interparticle forces that may be present, it is well known that a thin viscous film can exert an appreciable resistance to the deformation and relative motion of adjacent bounding surfaces, giving rise to forces of the type encountered in hydrodynamic lubrication.

In an exceedingly interesting previous work, which has also been commented upon by Batchelor [3], Frankel and Acrivos [1] have, in effect, employed the notion of a lubricated contact zone, in their theoretical treatment of the viscosity of highly concentrated suspensions of rigid spheres. In this way, they were able to provide an asymptotic, high-concentration theory for the classical Einstein problem and, thereby, to account theoretically for the experimental observations of an ultimate sharp increase of viscosity with particle concentration, an effect which had previously been described only by empirical formulae and perhaps attributed erroneously to mechanical contact of the particles.

On the other hand, in the area of solid mechanics, there is some interest in the mechanical behavior of deformable granular media, consisting of packed beds of solid particles in intimate mechanical contact with one another. 
Deresiewicz [4] has given an admirable review of this subject, with reference to the classical work of Reynolds [5] on rigid particles, but devoted primarily to the later works of Mindlin [6,7], and others, on elastic particles and the allied problem of "Hertzian" contact modified by surface friction.

The present work is concerned with systems which possess a certain similarity to both those just discussed. In the present case, we assume that the typical nearest distance separating neighboring particles is small but non-zero. That is, the minimum thickness of the interstitial liquid phase $h$, say, is assumed to be much smaller than the typical linear dimension or the typical radius of curvature of the adjacent particle surfaces $R$, say. Thus, as suggested by the work of Frankel and Acrivos [1], we shall adopt the classical (Reynolds) theory of hydrodynamic lubrication to describe the viscous flow and stress generated in the neighborhood of points of near contact, for $h / R \rightarrow 0$.

Furthermore, it is our hypothesis that, to terms of leading order in $h / R$, the regions of near contact account for all the effect of the interstitial liquid; i.e. that for $h / R \rightarrow 0$, these regions serve to transmit essentially all the stress through the suspension, as it were, from particle to particle, exactly as with the points of contact in a "dry" packed bed.

Although one may formulate certain criticism of the classical lubrication approximation to be employed here, it appears, on the basis of works like those of Cooley and O'Neill [8], and O'Neill and Stewartson [9], that this approximation can be regarded as the lowest-order term, for $h / R \rightarrow 0$, of a singular-perturbation theory and, as such, is amenable to higher-order improvements.

Within the framework of the lowest-order terms, i.e. the classical lubrication theory, we shall be concerned here with the deformation of the suspended particles under the combined action of an applied macroscopic deformation of the suspension and the viscous forces of the intervening liquid films. Because of the assumed deformability of the particles, we encounter then a problem involving the hydrodynamic lubrication of compliant surfaces, a problem which arises in several other contexts and which for the case of Hookean elastic bodies is governed by the so-called "elastohydrodynamic" equations (cf. Christensen [10]).

Our objective here is to consider the simplest formulation of this kind of problem, in the context of our idealized suspension model and, further, to consider certain parametric limits and the implications for suspension rheology. To this end, we shall treat only the most elementary case, that of equal-sized Hookean solid spheres suspended in an incompressible Newtonian liquid, with interparticle force fields, buoyancy forces and inertial effects all being negligible.

\section{Equations for the near-contact region}

Here, we wish to set down the relations which will be assumed to govern the shape of the deformable particle surfaces and the stresses developed in the 
region of near contact, under the action of a macroscopic deformation of the suspension.

Given any representative pair of adjacent particles having an initially spherical shape, we assume that their relative motion and deformation under an applied macroscopic velocity gradient $\dot{L}=(\nabla v)^{T}$ will consist of a systematic or "mean" homogeneous motion, representative of the suspension as a whole, plus a "local" non-homogeneous deformation and motion, to some extent random, and representative of the local arrangement and interaction of particles.

The systematic motion is assumed to consist of the following constituent motions: (1) a relative motion of the centers of the spheres, given by the applied velocity gradient as

$\Delta v=v_{1}-v_{2}$

with

$v_{1}=\dot{L} \cdot x_{1}, v_{2}=\dot{L} \cdot x_{2}$

and with $x_{1}, v_{1}, x_{2}, v_{2}$ denoting the position and velocity vectors of the respective sphere centers; and (2) a homogeneous deformation of the spheres, identical for each, and representative of the mean displacement gradient $\boldsymbol{L}_{s}$ or the associated velocity gradient $\dot{L}_{s}$ of the solid phase. This corresponds to velocity fields of the form

$v=\dot{L}_{s} \cdot x+v_{i}, i=1,2$

inside spheres 1 and 2 , respectively, where $v_{1}, v_{2}$ are given by eqn. (1). In the case of rigid particles treated by Frankel and Acrivos [1], $\dot{L}_{s}+\dot{L}_{s}^{T}$ vanishes identically.

The local deformation and motion referred to above consist, then, of the remaining material motion. This includes particle deformations which are nonhomogeneous, owing to the localized nature of contact forces, and which are random, to the extent that the spatial distribution of contact zones is random; and it includes relative motions of neighboring particle centers, which differ from the postulated mean motion, because of particle interactions.

It is, of course, precisely this localized particle motion and interaction that makes for difficulty in the kinetic theory of the suspension, an aspect of the subject that is common to molecular kinetic theories of dense phases. In addressing this problem the best we can do, short of attempting a comprehensive statistical mechanics, is to limit our attention to the mean motion and the associated local particle deformation resulting from some assumed arrangement of neighboring particles (cf. the discussion of Batchelor [3] and Frankel and Acrivos [1]), a point which we shall reconsider in Sections 3 and 4 below.

As for the mean motions, one sees that the combined action of (1) and (2) would in general produce from a pair of neighboring spherical surfaces a pair of congruent, identically oriented, and possibly intersecting ellipsoidal surfaces, as illustrated in Fig. 2. While we shall presently employ such configura- 
173
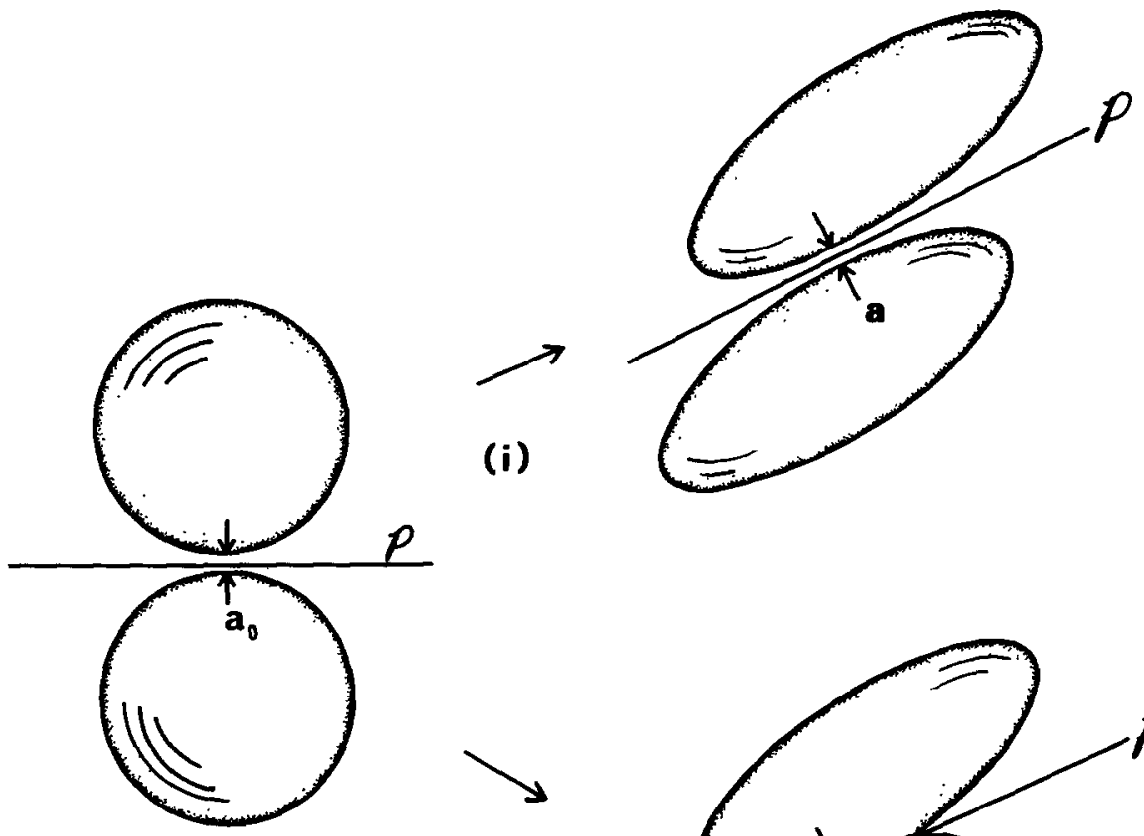

(ii)

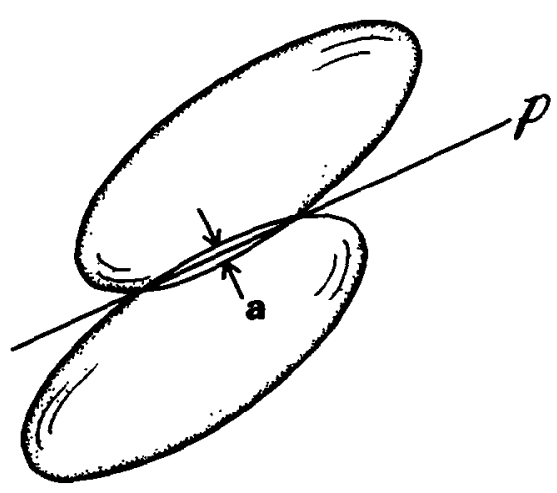

Fig. 2. Pair of ellipsoidal surfaces and produced from a pair of neighboring spheres by a relative translation of their centers, together with an identical deformation of each. An edge of the midplane $\mathcal{P}$ normal to the line of extremal approach $a$ is denoted by $\mathcal{P}$. (i) For no intersection, $a$ denotes the minimum distance of separation and (ii) for intersection, $a$ denotes the maximal distance of separation inside the region of intersection.

trons to define a local system of coordinates in the contact zone, we anticipate an associated local particle deformation, referred to in the last paragraph, which serves to prevent actual physical contact as illustrated in Fig. 3. At any rate, all the solid strains arising from the above deformations are assumed to be infinitesimal in keeping with the assumption of Hookean elasticity, although the associated particle rotations may be finite.

Given, then, any pair of slightly deformed spheres in near contact, we consider the mid-plane normal to a line segment defining the distance of extrema approach, not for the actual particle surfaces but for the ellipsoidal surfaces resulting from the motions (1) and (2) above. In the case of non-intersecting ellipsoids, this line segment represents the distance of closest approach of the surfaces, whereas in the case of intersection it represents the maximum dis- 


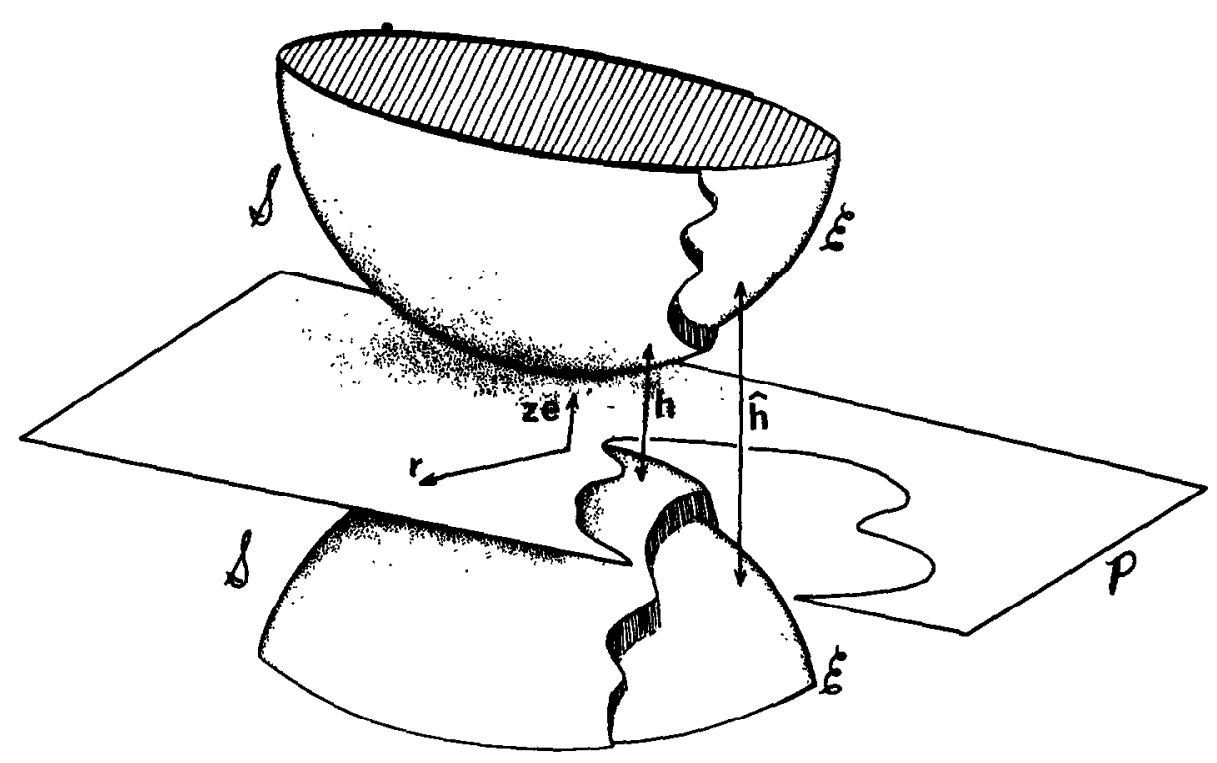

Fig. 3. Illustration of neighboring particles, showing the actual particle surfaces $\delta$, the hypothetical ellipsoidal surfaces $\mathcal{E}$, the corresponding separation distances $h(r)$ and $\hat{h}(r)$ the midplane of separation $\mathcal{P}$ with normal $e$ and associated cartesian coordinates $z$ and $r=x i+y j$.

tance between the surfaces inside the region of intersection.

Such a line segment can be chosen as the $z$-axis of a locally defined cartesian system with, say, $z=0$ defining the mid-plane, which we shall call the "contact plane". Then

$r=x i+y j$

denotes the position vector in the contact plane, with $z_{1}(r, t) z_{2}(r, t)$ defining the nearby particle surfaces, and

$h=h(r, t)=\left|z_{1}(r, t)-z_{2}(r, t)\right|$

representing the thickness of the liquid film.

Figure 3 illustrates schematically the actual particle surfaces and film thickness, together with those corresponding to the states of particle motion and deformation discussed above. We are interested here in the region of near contact, where the gap widths corresponding to the initial state and to the mean motion are given respectively by

$h_{0}=a_{0}+b_{0} r^{2}+\mathrm{O}\left(b_{0}^{3} r^{4}\right)$

and

$$
\begin{aligned}
\hat{h}(r, t) & =a(t)+r \cdot B(t) \cdot r+\mathrm{O}\left(b^{3} r^{4}\right) \\
& \equiv a(t)+b_{11} x^{2}+2 b_{12} x y+b_{22} y^{2}+\mathrm{O}\left(b^{3} r^{4}\right) .
\end{aligned}
$$


Here, the $a$ 's denote the corresponding extremal distances of approach and the $b$ 's are related to the local geometric curvature of the particle surfaces. In particular,

$b_{0}=1 / R_{0}$

where $R_{0}$ is the radius of the undeformed sphere, whereas

$$
3=b_{11} i i+b_{12}(i j+j i)+b_{22} \ddot{j},
$$

so that

$\operatorname{det} B \equiv b_{11} b_{22}-b_{12}^{2}=1 / R_{1} R_{2}$, and $\operatorname{tr} B \equiv b_{11}+b_{22}=\frac{1}{R_{1}}+\frac{1}{R_{2}}$

are the Gaussian curvature and twice the mean curvature, expressed in terms of $R_{1}(t)$ and $R_{2}(t)$, the principal curvatures associated with the mathematical surface $z=\hat{h}(r, t)$.

In terms of the parameters in (5), the present analysis is based on the assumption that quantities like

$\epsilon \equiv a_{0} b_{0}=a_{0} / R_{0}$

are much smaller than unity. The corresponding time-dependent parameters $a$ and $b$ in the expression of (5) for film thickness, which take on the values

$a=a_{0}, b_{11}=b_{22}=b_{0}, b_{12}=0$

at the initial state $t=0$, are in principle determined for $t>0$ by the associated macroscopic and homogeneous deformations of the suspension and the neighboring particle surfaces. Indeed, by means of (1) and (2) together with the assumption of small particle strain and some straightforward geometric considerations, relating to the geometry of the relevant ellipsoidal surfaces, one can obtain the following explicit differential equation for $\hat{h}$ :

$$
\begin{aligned}
& \frac{\partial \hat{h}}{\partial t}=\alpha(t)+\beta(t) \hat{h} \\
& \frac{\mathrm{d} a}{\mathrm{~d} t}=\alpha(t)+\beta(t) a, \quad \frac{\mathrm{d} b_{i j}}{\mathrm{~d} t}=\beta(t) b_{i j}
\end{aligned}
$$

where

$$
\begin{aligned}
\alpha(t) & =e \cdot\left(\dot{L}-\dot{L}_{s}\right) \cdot\left(x_{2}-x_{1}\right) \\
& =2 R_{0} e \cdot\left(\dot{E}-\dot{E}_{s}\right) \cdot e\left\{1+\mathrm{O}(\epsilon)+\mathrm{O}\left(E_{s}\right)+\mathrm{O}\left(\left[\dot{L}-\dot{L}_{s}\right] \cdot E_{s}\right)\right\}
\end{aligned}
$$

and

$\beta=e \cdot \dot{E}_{s} \cdot e$

with $e$ denoting the unit normal to the plane of contact. Here, as in the fol- 
lowing we denote, respectively, by expressions like

$E=E(t)=\frac{1}{2}\left(L+L^{T}\right), \dot{E}=\dot{E}(t)=\frac{1}{2}\left(\dot{L}+\dot{L}^{T}\right)$,

the (infinitesimal) strains and the rate of deformation derived from the macroscopic gradients $L$ and $L_{s}$ or $\dot{L}$ and $\dot{L}_{s}$. Then, the unit vector $e$ and $d=\left(x_{1}-\right.$ $\left.x_{2}\right) /\left|x_{1}-x_{2}\right|$ are determined by

$e=\left\{1+2(1-d d) \cdot E_{s}+O\left(E_{s}^{2}\right)\right\} \cdot d$

and

$\frac{\mathrm{d}}{\mathrm{d} t}\left(x_{1}-x_{2}\right)=\dot{L} \cdot\left(x_{1}-x_{2}\right)$

Of interest here is the actual particle deformation and the associated film thickness $h$ of (4), or, equivalently, the incremental particle deformation and the corresponding incremental film thickness $h--\hat{h}$. The latter quantities are attributed in the present context to fluid stress in the regions of near contact over and above that required to produce the mean macroscopic particle strain $\boldsymbol{E}_{s}$.

It is proposed here to employ the equations of hydrodynamic-lubrication theory, to determine the mean stress in the particle arising from the mean macroscopic motions and the action of the lubricated contact zones, as well as the incremental particle stress and strain in the vicinity of the individual contact zones.

Thus, we assume that in a representative particle the homogeneous strain corresponding to the macroscopic gradient $\dot{L}_{s}$, is sustained by a set of points of near-contact, distributed over the surface of the particle in a statistically representative way. The general formula

$T_{s}=\frac{1}{V} \iint_{A} n \cdot T x \mathrm{~d} A$

(cf. Brenner [2], Goddard and Miller [11]) gives the volume average stress in an inertialless, body-force free medium of volume $V$, in terms of the "dyadic" spatial moment of surface traction $\boldsymbol{n} \cdot \boldsymbol{T}$ over its bounding surface. Then, for one of our representative particles, (13) can be reduced to a finite summation over a discrete set of contact points, having the form

$T_{s}=\frac{1}{V} \sum_{i} f_{i} x_{i}\left\{1+O\left(l / R_{0}\right)\right\}$.

Here, $f_{i}$ represents the total force acting over a small region of near-contact, $l$, to be defined further below, is a typical linear dimension of this region, which we call a "contact zone", and $\boldsymbol{x}_{i}$ is a position vector representing the location of this region. In the case of slightly deformed spheres, eqn. (14) can be further reduced to 


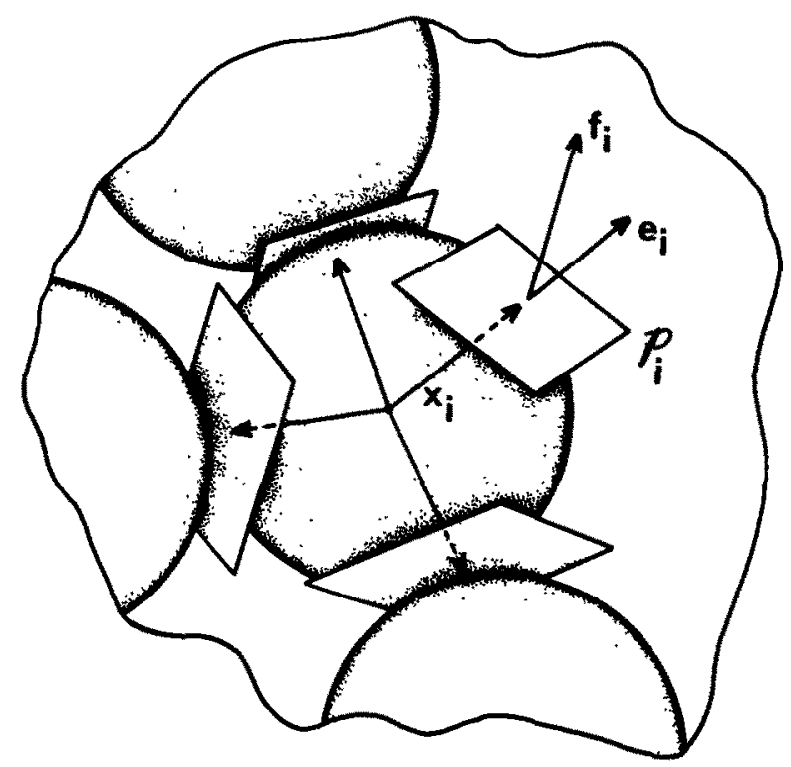

Fig. 4. Illustration of contact zones for a typical particle, showing the associated midplanes $\mathcal{P}_{i}$, a midplane normal $e_{i}$, a position vector $x_{i}$ and a resultant force vector $f_{i}$.

$T_{s}=\frac{3}{4 \pi R_{0}^{2}} \sum_{i} f_{i} e_{i}\left\{1+\mathrm{O}\left(l / R_{0}\right)+\mathrm{O}\left(E_{\mathrm{s}}\right)\right\}$,

where $e_{i}$ denotes the unit normal to the contact plane for the $i$ th region of near-contact. The symmetry of the tensors given by any of the expressions (13)-(15) follows from the condition of vanishing couple on the solid. This condition, as applied to (15), and the condition of vanishing force on the particle take on the forms:

$\sum_{i} f_{i} \times e_{i}=0$ and $\sum_{i} f_{i}=0$

Associated with the expression (15), then, we have the mean particle strain $E_{s}$, related to $T_{s}$ by the Hookean-elastic constitutive relation, together with the expression for the local surface traction $n \cdot T_{s}$.

The "contact" forces $f_{i}$ in (14)-(16) are to be obtained here by the appropriate equations of elastohydrodynamics. The first of these is a "Reynolds" equation, which connects the pressure distribution $p(r, t)$ in the lubricated contact zone, to the thickness of the fluid film $h(r, t)$ :

$\frac{1}{12 \eta} \nabla_{0} \cdot\left(h^{3} \nabla_{0} p\right)=\frac{\partial h}{\partial t}+\nabla_{0} \cdot\left(\bar{v}_{0} h\right)$

where $\eta$ is the (constant) viscosity of the fluid and $\nabla_{0}$ and $\bar{v}_{0}$ are two-dimen- 
sional vectors, defined in the plane of contact by:

$\nabla_{0}=i \frac{\partial}{\partial x}+j \frac{\partial}{\partial y}$

and

$\bar{v}_{0}=i \bar{u}_{0}+j \bar{v}_{0}$,

where $\bar{v}_{0}$ represents the arithmetic-average velocity tangent to the fluid film, of the bounding solid surfaces, at $z=z_{1}(r, t), z=z_{2}(r t)$, respectively (cf. Christensen [10]).

In addition to (17), we have for the fluid shear stress exerted on the upper and lower bounding surfaces in a direction tangential to the film, the vector

$\tau_{0}= \pm h \nabla_{0} p+\eta \Delta v_{0} / h$

where the \pm signs correspond, respectively, to the lower or upper $(\mp z)$ surfaces of the liquid film and $\Delta v_{0}$ denotes the difference in velocities, again tangential to the film, of the upper and lower bounding surfaces. The first term is associated with a pressure-driven ("Hele-Shaw") flow and the second with a simple shear.

For the present application, we shall simply neglect the convection or "entrainment" term $\nabla_{0} \cdot\left(\bar{v}_{0} h\right)$ in (17), on the grounds that a non-vanishing velocity $\bar{v}_{0}$ involves either a mean translation of neighboring spheres or else involves terms $\mathrm{O}(h)$ which result from particle deformation, both of which are presumed small in their effect compared to the term $\partial h / \partial t$ in (17). (Cf. Frankel and Acrivos [1], who effectively adopt the same condition.)

The second set of equations connecting $p$ and $h$ are those customarily obtained by considering the local elastic deformation and, in particular, the normal displacements of the solid surface resulting from the boundary tractions exerted by the adjacent fluid. Within the usual elastohydrodynamic approximation, as in the analogous Hertzian contact problem (Timoshenko and Goodier [12], Love [13], Lubkin [14]), this problem is treated as an elastostatic boundary-value problem on the semiinfinite half-space, of the type solved by Boussinesq [15] for normal tractions specified on the bounding plane.

However, it appears that in the usual applications to lubrication problems the effect of any shearing tractions on the normal displacement is entirely neglected (Christensen [10], Bell and Kannel [16]), in distinct contrast to the treatments of solid contact with friction (Deresiewicz [4,19,20], Mindlin [6], Lubkin [7]). It seems, to this author, that for all but the special case of incompressible solids, for which there is no coupling between shearing and normal displacements, the neglect of such terms is subject to considerable criticism. While the terms in question can be rigorously incorporated into the usual elastohydrodynamic problem, by employing the expression for traction in (19), it appears that this would add considerably more difficulty to its treatment. Therefore, for the present analysis, attention will be restricted to 
the case of an incompressible Hookean solid.

The normal displacement of the boundary $(z=0)$ of an elastic half space $(z>0)$ subject to the normal pressure $p(r, t)$ on that boundary is given by

$w_{0}(r)=\frac{1}{4 \pi \mu^{\prime}} \iint_{A} \frac{p\left(r^{*}\right)}{\rho} d A\left(r^{*}\right)$,

with

$\rho=\rho\left(r, r^{*}\right) \equiv\left|r-r^{*}\right|=\sqrt{\left(x-x^{*}\right)^{2}+\left(y-y^{*}\right)^{2}}$

and

$\mu^{\prime}=\frac{E}{4\left(1-\nu^{2}\right)}=\frac{\mu}{2(1-\nu)}$,

where $E=2(1+\nu) \mu, \mu$, and $\nu$ denote, respectively, the Young's modulus, Lamé shear modulus and Poisson's ratio for the solid. As indicated above, eqn. (20) is valid for a surface free from shearing traction or, otherwise, for an incompressible solid, in which case $\nu=1 / 2$ and $\mu^{\prime}=\mu$, as is assumed here.

In lieu of applying (20) to the total deformation and stress for the solid particles, we may equally well apply it to the incremental deformation and stress, over and above that of the mean macroscopic deformation of (2). Thus, by considering the incremental displacement of both the upper and lower solid surfaces bounding the liquid film, we obtain, as a variation on the usual elastohydrodynamic equation for the film thickness $h(r, t)$,

$h(r, t)=\hat{h}(r, t)+\frac{1}{2 \pi \mu} \iint_{A} \frac{p\left(r^{*}, t\right)-\hat{p}\left(r^{*}, t\right)}{\rho} \mathrm{d} A$,

where $\hat{h}(r, t)$ is identical with the film thickness of (5) and (10) resulting from the homogeneous particle deformation (2), while $\hat{p}$ is to be taken equal to the arithmetic average of the corresponding elastic normal pressure at the adjacent particle boundaries.

In the case of the Hookean solid at hand, the boundary pressure associated with the homogeneous deformation (2) is given by

$p_{s}(r, t)=-n \cdot T_{s} \cdot n=-2 \mu n \cdot E_{s} \cdot n$,

where $n(r, t)$ denotes the unit normal at the boundary point in question. In principle, the specification of $\boldsymbol{n}$ for both of the particle surfaces bounding our liquid film would require a knowledge of the individual surface shapes $z_{1}(r, t)$, $z_{2}(r, t)$, and not just of $h(r, t)$ alone. However, it appears consistent with the approximation of the flat boundary in (19) and (21) to take $n= \pm e$, at the lower and upper surfaces respectively, with $e$ denoting, as above, the normal to the contact plane. In this case, $\hat{p}$ can be taken simply to have the uniform value

$\hat{p}=-e \cdot T_{s} \cdot e=-2 \mu e \cdot E_{s} \cdot e$ 
independent of $r$ in the region of near contact.

In any event, given an assumed set of homogeneous deformations in (1) and (2), and the associated functions $\hat{h}(r, t) \hat{p}(r, t)$, we are interested in solutions $h(r, t), p(r, t)$ to the elastohydrodynamic equations (17) and (21) which will exhibit an appropriate behavior at the periphery of the near-contact region.

Owing to its method of derivation, we may expect (17) to be valid only in a region $r=|r| \leqslant O(l)$ for $\epsilon \rightarrow 0$, where

$a_{0}<<l<<R_{0}$

and, based on the previous works of O'Neill et al. [8,9], we expect further that $l / R_{0}=\mathrm{O}\left(\epsilon^{1 / 2}\right)$. Whatever the magnitude of the characteristic length $l$, we can introduce a characteristic magnitude of the macroscopic deformation rate, say $\dot{e}$, and upon employing dimensionless variables

$r / l, h / a_{0}, l p / a_{0} \mu, \dot{e} t$

which we shall denote by the same symbols as their dimensional counterparts, we may express the elastohydrodynamic equations (17), with $\bar{v}_{0}=0$, and (21) in the dimensionless form

$\nabla_{0} \cdot\left(h^{3} \nabla_{0} p\right)=\lambda \frac{\partial h}{\partial t}$,

$h-\hat{h}=\frac{1}{4 \pi} \iint_{A} \frac{p\left(r^{*}, t\right)-\hat{p}\left(r^{*}, t\right) d A}{\rho}$,

where

$\lambda \equiv 12\left(\frac{l}{a_{0}}\right)^{3} \frac{\eta \dot{e}}{\mu}$.

The parameter $\lambda$ will be seen to represent a characteristic "relaxation" time for the suspension, which is rendered dimensionless by $\dot{e}$.

We are interested here in solutions $h, p$ to (26) such that

$p-\hat{p} \rightarrow 0$ and $h-\hat{h} \rightarrow 0$, for $r \rightarrow \infty$.

Given a general solution to (26), one could obtain a solution for the macroscopic particle stress $T_{s}$ corresponding to a set of assumed gradients $\dot{L}, \dot{L}_{s}$ as follows: First, one can cast (13) into the form

$T_{s}+T_{s}: \chi=\frac{1}{V} \iint_{A}\left[-\left(p-p_{s}\right) n+\tau\right] x \mathrm{~d} A$

where $p_{s}$ is given by (22), the shear stress on the particle surface by

$\tau=(1-n n) \cdot T \cdot n$

and $\chi$ is a fourth-rank tensor defined by 
$\chi \equiv \frac{1}{V} \iint_{A} n n n x \mathrm{~d} A$.

Then, employing relations like those of (14), (15), and (23), one obtains

$T_{s}+T_{s}: \chi=\sum_{i}\left\{-P_{i} e_{i} e_{i}+\tau_{i} e_{i}\right\}$

where

$\chi=\frac{3}{4 \pi} \iint_{A^{\prime}} n n n n \mathrm{~d} A\left\{1+\mathrm{O}\left(E_{s}\right)\right\}$

and

$P_{i}=\frac{3}{4 \pi R_{0}^{2}} \iint_{A_{i}}(p-\hat{p}) \mathrm{d} A\left\{1+\mathrm{O}\left(E_{s}\right)+\mathrm{O}\left(l / R_{0}\right)\right\}$,

with $A^{\prime}$ and $A_{i}$ denoting, respectively, integrals over the unit sphere and over the $i$ th contact plane. The quantity $\tau_{i}$ represents the shear stress contribution from the $i$ th contact plane and involves an integral of the form

$\tau_{i}=\frac{3}{4 \pi R_{0}^{2}} \iint_{A_{i}} \tau_{0} \mathrm{~d} A$

where $\tau_{0}$ has the form (19). Here, we expect the first term in (19), involving $\nabla_{0} p$, to give a finite contribution to the integral (30), when taken over an infinite contact plane $A_{i}$, and whenever $p \rightarrow \hat{p}$, a constant, for $r \rightarrow \infty$. On the other hand, we expect the second term in (19) $\Delta v_{0} / h$ to involve a logarithmically divergent integral, since $h \simeq r^{2}$ for $r \rightarrow \infty$. This, however, does not represent a fundamental singularity, for, as mentioned earlier, the lubrication approximation on which (30) is based is valid only in a region $0 \leqslant r \leqslant \mathrm{O}(l)$ and must be replaced by an appropriate "outer" approximation for $r \rightarrow \infty$.

Thus, in effect, the integral in (29) should be taken over a region bounded by a circle $r=\mathrm{O}(l)$. As evident from the previous works of Frankel and Acrivos [1] and O'Neill et al. [8,9], we expect that this will give rise to shear stress contributions of, at most, $\mathrm{O}(\log \epsilon)$, for $\epsilon \rightarrow 0$, and that these will be of a smaller order in $\epsilon$ than the pressure contribution $P_{i}$ in (29).

Then, making use of known formulae (Brenner and Condiff [17]) such as

$\frac{1}{4 \pi} \iint_{A^{\prime}} n_{i} n_{j} \mathrm{~d} A=\frac{1}{3} \delta_{i j}$

and

$\frac{1}{4 \pi} \iint_{A^{\prime}} n_{i} n_{j} n_{k} n_{l} \mathrm{~d} A=\frac{1}{15}\left(\delta_{i j} \delta_{k l}+\delta_{i k} \delta_{j l}+\delta_{i l} \delta_{j k}\right)$ 
in particular the latter to evaluate the integral in (29), we may express (29) in the deviatoric form

$2 \mu E_{s}=T_{s}-\frac{1}{3}\left(\operatorname{tr} T_{s}\right) 1 \doteq \frac{5}{3} \sum_{i} P_{i}\left(\frac{1}{3} 1-e_{i} e_{i}\right)$,

which is the only relevant stress for the case of the incompressible solid. In addition to error terms of the kind indicated in conjunction with (29), eqn. (32) will also involve a relative error $\mathrm{O}((1 / P) \log \epsilon)$, where $P$ denotes a quantity which is $O\left(P_{i}\right)$ for $\epsilon \rightarrow 0$. As will be established below, for the case of slightly deformable particles at hand $P_{i}=\mathrm{O}(1 / \epsilon)$ and, hence, this error term is $O(\epsilon \log \epsilon)$ for $\epsilon \rightarrow 0$.

Within the framework of the preceding approximation, the condition of vanishing couple in (16) is satisfied and the condition of vanishing force in (16) becomes

$$
\sum_{i} P_{i} e_{i}=0
$$

To the order of terms considered in (32) and (33) we should like, then, to have solutions of (26), to permit evaluation of the normal force contribution $P_{i}$ from the $i$ th contact zone. Since the functions $\hat{h}, \hat{p}$ in (26) depend on the particle deformation (or deformation rate) and stress, so does $P_{i}$; therefore eqn. (32) provides a relation between the macroscopic deformation of the suspension and the particle stress or strain.

The strong non-linearity in (26) appears to rule out all but the most special analytical solutions $t$. Therefore, it is worthwhile to consider asymptotic solutions of (28) for extreme values of the parameter $\lambda$. Here, we shall focus attention on the limit of the nearly rigid particle, $\lambda \rightarrow 0$.

\section{Nearly rigid particles and suspension mechanics}

The limit $\lambda \rightarrow 0$ is, strictly speaking, the appropriate one for the present discussion, where we have already assumed a small particle deformation. In the limit $\lambda \rightarrow 0$, we assume the solution to (26) can be represented as the perturbation series

$h=\hat{h}+\lambda h^{(1)}+\mathrm{O}\left(\lambda^{2}\right)$

$p=\hat{p}+\lambda p^{(1)}+\mathrm{O}\left(\lambda^{2}\right)$

taking $\hat{p}$ to be an arbitrary constant, as is appropriate to the rigid particle limit, where particle stress is rheologically indeterminate.

$\dagger$ In this regard, we note that Christensen [10] has resorted to numerical methods to treat a related "elastohydrodynamic squeeze-film" problem. 
Then, it follows that $h^{(1)}$ and $p^{(1)}$ satisfy

$\nabla_{0} \cdot \hat{h}^{3} \nabla_{0} p^{(1)}=\frac{\partial \hat{h}}{\partial t}$

and

$h^{(1)}(r, t)=\frac{1}{4 \pi} \iint \frac{p^{(1)}\left(r^{*}, t\right) \mathrm{d} A^{*}}{\rho\left(r^{*}, r\right)}$.

In principle, one can solve the first of these relations, eqn. (35), for $p^{(1)}$, in terms of $\hat{h}$, before proceeding to the second, for $h^{(1)}$, with a similar kind of scheme being evident for higher-order terms. For the problem at hand, $\hat{h}$ is given by (5) and (10), which, when expressed in terms of dimensionless variables consistent with (25), take on similar forms, but now with

$\frac{\partial \hat{h}}{\partial t}=\frac{\alpha}{\epsilon}+\beta \hat{h}$,

or

$\frac{\mathrm{d} a}{\mathrm{~d} t}=\frac{\alpha}{\epsilon}+\beta a, \quad \frac{\mathrm{d} B}{\mathrm{~d} t}=\beta B$, etc.

The variables appearing here are defined in terms of their dimensional counterparts by (25) and by the additional expressions

$a / a_{0}, l^{2} b_{i j} / a_{0}, \alpha / \dot{e} R_{0}, \beta / \dot{e}$.

It will now be shown that one may obtain an exact solution to (35) for $p^{(1)}$ whenever $\hat{h}$ is given by (5), with $a(t)$ and $\boldsymbol{B}(t)$ being arbitrary functions of time.

Thus, as a variant of the related lubrication formula of Kapitza [18], we have the following lemma: A particular solution $p$ to (35), when $\hat{h}$ is given by the quadratic form in (5), with $\operatorname{tr} B=b_{11}+b_{22}>0$, is

$p=(b+r \cdot A \cdot r) / \hat{h}^{2}$,

where

$A=-\frac{1}{4}(\dot{B}+\xi B) \cdot K$,

$b=-\frac{1}{4}(\dot{a}+\xi a) / \operatorname{tr} B$,

with

$\xi=\frac{\operatorname{tr}(\dot{B} \cdot K)}{1-\operatorname{tr}(B \cdot K)}$,

$K=\left[B+(\operatorname{tr} B) 1_{0}\right]^{-1}$,

and

$\dot{B} \equiv \mathrm{d} B / \mathrm{d} t, \quad \dot{a}=\mathrm{d} a / \mathrm{d} t, \quad 1_{0}=i i+j i$ 
the idemfactor $1_{0}$ and the other tensor quantities $A, K$ being defined, as with $B$, on the two-dimensional vector space of the contact plane. This result may be verified directly by substitution of (39) into (35). We recall that the quantity $\operatorname{tr} B$ is the dimensionless form of the curvature in eqn. (7).

Although the general solution to (35) involves an additive solution to the homogeneous equation, obtained by setting $\partial \hat{h} / \partial t=0$, it appears that the particular solution (39) suffices for the present application, since it satisfies the boundary condition (27). Furthermore, in the special case of interest here, where (37) holds as well, one can further reduce the form of (39). Thus, for $\epsilon \rightarrow 0$, such that the term $\alpha / \epsilon$ dominates in (37), one obtains

$p=-\frac{\alpha}{4(\operatorname{tr} B) \epsilon \hat{h}^{2}}[1+O(\epsilon)]$

The terms denoted by $O(\epsilon)$ in (40) involve coefficients which behave like $r^{2}$ for large $r$, so that (40) may be considered valid only in a region $r^{2}=\mathrm{O}(1 / \epsilon)$ for $\epsilon \rightarrow 0$. Moreover, when it comes to evaluation of the normal stress $P_{i}$ in (29), the terms denoted by $O(\epsilon)$ in $(40)$ would give rise to logarithmically divergent integrals, since

$\frac{r^{2}}{\hat{h}^{2}} \times r \mathrm{~d} r \simeq$ const. $\frac{\mathrm{d} \hat{h}}{\hat{h}}$

for $r \rightarrow \infty$ and $\hat{h} \rightarrow \infty$. This is precisely the type of singularity associated with the shear stress $\tau_{i}$, arising from (19) together with (30), and, once again, indicates the non-uniform asymptotic character of the lubrication approximation at the periphery of the lubrication zone. However, the terms denoted by $O(\epsilon)$ in (40), like the term $\tau_{i}$ in (30), will give rise to contributions $O(\epsilon \ln \epsilon)$ which are negligible compared to the leading terms retained here for $\epsilon \rightarrow 0$.

For the nearly rigid particle $\lambda \rightarrow 0$ we obtain, then, from (40), (34), (29) and (25), that

$$
\begin{aligned}
P_{i} & =\frac{3 l^{2}}{4 \pi R_{0}^{2}}\left(\frac{\mu a_{0}}{l}\right) \lambda \iint p^{(1)} \mathrm{d} A\{1+\mathrm{O}(\lambda)\} \\
& =\frac{3 \mu}{16 \pi}\left(\frac{l}{R_{0}}\right)^{2}\left(\frac{a_{0}}{l}\right) \frac{\lambda \alpha_{i}}{\epsilon \operatorname{tr} B} \iint_{-\infty}^{+\infty} \frac{\mathrm{d} x \mathrm{~d} y}{\hat{h}^{2}}\{1+\mathrm{O}(\epsilon \ln \epsilon)+\mathrm{O}(\lambda)\}
\end{aligned}
$$

where the integral is understood to be expressed in terms of dimensionless geometric variables, according to (25). Since $\hat{h}$ has the form (10), we may make use of the formula

$$
\int_{-\infty}^{+\infty} \frac{\mathrm{d} x \mathrm{~d} y}{(a+r \cdot B \cdot r)^{2}}=\frac{\pi}{a}(\operatorname{det} B)^{-1 / 2}
$$

the preceding relation, and the geometric relations (7) to conclude that, with 
relative error $O(\lambda)+O(\epsilon \log \epsilon)$,

$P_{i}=\frac{9}{2}\left[\frac{\left(R_{1} R_{2}\right)^{3 / 2}}{\left(R_{1}+R_{2}\right) R a_{0}}\right]_{i} \eta e_{i} \cdot\left(\dot{E}-\dot{E}_{s}\right) \cdot e_{i}$

which has been recast here in terms of dimensional quantities associated with the $i$ th point of contact. Thus, $P_{i}$ is $\mathrm{O}(1 / \epsilon)$ for $\epsilon \rightarrow 0$, indicating that the shear stress terms $\tau_{i}$ in (29) are $O(\epsilon \log \epsilon)$ relative to the normal stress $P_{i}$.

By means of (41), (32) and (33) can be expressed as

$\sum_{i} \theta_{i} e_{i} \cdot\left(\dot{E}-\dot{E}_{s}\right) \cdot e_{i} e_{i}=0$

and

$E_{s}=-\sum_{i} \theta_{i}\left(\frac{1}{3} 1-e_{i} e_{i}\right) e_{i} \cdot\left(\dot{E}-\dot{E}_{s}\right) \cdot e_{i}$,

where

$\theta_{i}=\frac{15}{4}\left[\frac{\eta\left(R_{1} R_{2}\right)^{3 / 2}}{\mu\left(R_{1}+R_{2}\right) R_{0} a}\right]_{i}$.

Again, the subscript $i$ refers to the $i$ th point of contact, with $\theta_{i}$ denoting a characteristic time parameter.

We recall that (42) represents a force balance, while (43) provides a relation between the mean particle strain and the imposed macroscopic strain rate. These relations are complicated by their dependence on the distribution of points of near contact, that is the distribution of the unit vectors $\boldsymbol{e}_{i}$, and by dependence of the time parameter on the particle strain and the position of neighbors through $R_{1}(t), R_{2}(t), a(t)$, which are in turn governed by (36). Consistent with our assumption of small particle strain, or small $\lambda$, the curvature terms $R_{1}, R_{2}$ may be replaced by $R_{0}$ which gives, in lieu of the corresponding relation in (42),

$\theta_{i}=\frac{15}{8} \frac{\eta}{\mu} \frac{R_{0}}{a_{i}}$

with a relative error $\mathrm{O}\left(E_{s}\right)$ or $\mathrm{O}(\lambda)$. However, this relation still involves $a_{i}(t)$, which is to be determined from

$\frac{\mathrm{d} a_{i}}{\mathrm{~d} t}=2 R_{0} e_{i} \cdot\left(\dot{E}-\dot{\boldsymbol{E}}_{s}\right) \cdot e_{i}$

derived from (37) and (10).

The determination of mean particle strain $E_{s}(t)$ according to (43), (44) and (45) requires a specification of the number of contact points and the associated directions $e_{i}(t)$, which should be regarded as random variables described by an appropriate joint probability distribution. By means of (45), this distribu- 
tion would lead to a joint distribution of the $\boldsymbol{e}_{i}$ and the spacings $\boldsymbol{a}_{i}$.

In principle, these distributions are governed by the suspension dynamics and are subject not only to equations like (42), describing nearest neighbor forces, but also to longer-range effects. Given the general status of other kinetic theories of concentrated phases, we should not expect to make much progress on the exact determination of the distribution functions.

This difficulty is evident in the previous analysis of Frankel and Acrivos [1] and, as they have done, we might choose to regard the number of nearest neighbors of a representative particle, the analogue of the "coordination number" in molecular theories, to be given and determined mainly by particle concentration. Then it will be noted that eqns. (43) and (45) merely require for their treatment the marginal probability distribution of individual contact directions $e_{i}$. Thus, (45) leads to a joint distribution $f(e, a, t)$, giving in the usual way the probability density of finding a point of near contact in a region $\mathrm{d}^{2} e(t) \mathrm{d} a$, where $\mathrm{d}^{2} e$ denotes a differential solid angle. Denoting averages with respect to this distribution by brackets \{\rangle , we may express the averaged form of (43) as

$E_{s}=-\frac{N}{3}[1\langle\theta e e\rangle-3\langle\theta e e e e\rangle]:\left(\dot{E}-\dot{E}_{s}\right)$,

where $N$ denotes the number of nearest neighbors of a representative particle, and where $\theta=\theta_{i}$ is defined in terms of $a=a_{i}$ as in (44).

Again, an exact determination of the distribution function required in (47) is not expected to be readily forthcoming, although certain reasonable approximations may suggest themselves.

For example, one might assume that $\theta(a)$ in (47) can be replaced by the constant

$\theta=\frac{15}{8} \frac{\eta}{\mu} \frac{R_{0}}{\langle a\rangle}$,

where $\langle a\rangle$ is governed by the averaged form of (46)

$\frac{\mathrm{d}\langle a\rangle}{\mathrm{d} t}=2 R_{0}\langle e e\rangle:\left(\dot{E}-\dot{E}_{s}\right)$

The resulting averages $\langle e e\rangle$ and $\langle e e e e\rangle$ in (47) and (48) can then be evaluated by means of an assumed distribution.

By way of illustration, if one assumes the (time-independent) isotropic distribution $f(e)=1 / 4 \pi$, then, with the aid of (31), eqn. (48) becomes $\frac{\mathrm{d}\langle a\rangle}{\mathrm{d} t}=2 R_{0} \operatorname{tr}\left(\dot{E}-\dot{E}_{s}\right)=0$,

the second equality following from the assumed incompressibility of the medium. Therefore,

$\langle a\rangle=a_{0}$ and $\theta=\theta_{0} \equiv \frac{15}{8} \frac{\eta}{\mu} \frac{R_{0}}{a_{0}}$, 
so that, again with the aid of (31), (48) becomes

$\bar{\theta} \dot{E}_{s}+E_{s}=\bar{\theta} \dot{E}$,

where

$\bar{\theta}=\frac{2 N}{15} \theta_{0}=\frac{N}{4} \frac{\eta}{\mu}\left(\frac{R_{0}}{a_{0}}\right)$

which represents the generally desired type of relation for the macroscopic solid strain in terms of the macroscopic deformation rate. Incidentally, we note that the force balance (42) is satisfied identically by the approximations used here, since $\langle e e e\rangle$ vanishes.

While other, more desirable approximations might have been introduced, eqn. (49) nevertheless serves to suggest a plausible type of constitutive equation for the suspension as well as an important relation between material parameters involved. In particular, we recall that the proper definition of macroscopic stress in a suspension is the volume average (Batchelor [3], Brenner [2]), so that for the concentrated suspensions at hand, eqn. (49) suggests a viscoelastic constitutive equation of the (Maxwell) form,

$\boldsymbol{T}+\bar{\theta} \dot{\boldsymbol{T}}=2 \bar{\eta} \dot{\boldsymbol{E}}$,

where

$\bar{\eta}=\phi \mu \bar{\theta}=\phi_{m} \mu \bar{\theta}\{1+\mathrm{O}(\epsilon)\}$

is an effective viscosity for the suspension, $\phi$ denoting the volume fraction of the solid particles. Here, $\phi$ is near to its "maximum" value $\phi_{m}$, which corresponds to $\epsilon=a_{0} / R_{0} \rightarrow 0$ and depends on the assumed type of nearest neighbor arrangement, or packing.

In the limit $\epsilon \rightarrow 0$, eqns. (50) and (51) suggest a Maxwell fluid, nearly Newtonian and having large viscosity $\bar{\eta}$ as well as a large relaxation time $\bar{\theta}$. In view of the assumption of nearly rigid spheres on which (51) is based, this is entirely plausible, and one might expect the indicated relation between $\bar{\eta}$ and $\bar{\theta}$ to be approximately valid for more general circumstances than we have considered here.

Although criticized by Batchelor [3] as not allowing for sustained flow, the type of rigid-sphere model of Frankel and Acrivos [1], or the variant considered here, may nevertheless be considered applicable to the initial suspension behavior near a state of rest, and also might serve to describe the smallamplitude oscillatory behavior of concentrated suspensions. In that regard, we recall that, based on various "cage" or "cell" models of the nearest neighbor configuration, Frankel and Acrivos [1] have taken

$\epsilon=2\left[\left(\frac{\phi_{m}}{\phi}\right)^{1 / 3}-1\right]$

for $\phi \rightarrow \phi_{m}$. Then, by their method of averaging the dissipation function for 
nearly touching spheres, they further obtain an effective viscosity

$\bar{\eta} / \eta=\frac{9}{8} \frac{\left(\phi / \phi_{m}\right)^{1 / 3}}{1-\left(\phi / \phi_{m}\right)^{1 / 3}}$

for rigid sphere suspensions with a simple cubical packing and, hence, $N=6$ nearest neighbors.

The equivalent result of the present work is given by (50) and (51) as

$\bar{\eta} / \eta=\frac{\bar{\theta} \phi_{m} \mu}{\eta}=\frac{N \phi_{m}}{8} \frac{\left(\phi / \phi_{m}\right)^{1 / 3}}{1-\left(\phi / \phi_{m}\right)^{1 / 3}}$

which for the simple-cubic array with $N=6, \phi_{m}=\pi / 6$ gives a factor $N \phi_{m} / 8=$ $\pi / 8$ instead of the factor $9 / 8$ in (53). On the other hand, for a cubical or hexagonal closest-packed array with $N=12, \phi_{m} \doteq 3 / 4$, one has $N \phi_{m} / 8 \doteq 9 / 8$ in accordance with (53), which, as shown by Frankel and Acrivos, is supported by experimental data. However, at this juncture it is not clear why the different methods of averaging yield such different results for the same packing of particles.

\section{Conclusions}

In summary, we have seen how the theory of elastohydrodynamics for the lubricated compliant contact can be adapted to the mechanics of concentrated suspensions, as an extension of the theory of Frankel and Acrivos [1]. For the case of slightly deformable Hookean spheres this leads, not surprisingly perhaps, to a constitutive equation of the Maxwell variety. However, one can extract quantitative predictions for the viscosity and relaxation time, which exhibit a similar dependence on particle concentration and a strong incrcase in the limit of touching particles, like that found by Frankel and Acrivos [1].

The present analysis indicates a way in which one may derive the complete stress tensor for such a system. For reasons which are as yet unclear to the author, this leads to a somewhat different estimate of viscosity than that of Frankel and Acrivos [1].

Neither analysis provides more than a cursory treatment of the statistical mechanics of the suspension, a subject which bears further study, to provide a more rigorous footing and perhaps to elucidate the discrepancies between the results. Furthermore, it would be interesting to consider the case of highly compliant solid particles, the opposite extreme of that considered here.

While it is questionable whether the present idealized model would be directly applicable to the most commonly found, real systems with a deformable microstructure, the analysis may point to a convenient way of describing the mechanics of particle interaction and deformation in such interesting real systems as emulsions and foams. Furthermore, in the case of solid dispersions, where the statistics of the particle arrangement can he specified in a more definite way, the techniques developed here may find extensive application. 


\section{References}

1 N.A. Frankel and A. Acrivos, On the viscosity of a concentrated suspension of solid spheres, Chem. Eng. Sci., 22 (1967) 847-853.

2 H. Brenner, Suspension rheology. In W.R. Schowalter (Ed.), Progress in Heat and Mass Transfer, Vol. 5, Pergamon Press, Oxford, New York, 1972, pp. 89-129.

3 G.K. Batchelor, Transport properties of two-phase materials with random structurc. In M. Van Dyke, W.G. Vincenti and J.V. Wehausen (Eds.), Annual Reviews of Fluid Mechanics, Vol. 6, Annual Reviews, Inc., Palo Alto, 1974, pp. 227-225.

$4 \mathrm{H}$. Deresiewicz, Bodies in contact with application to granular media. In Geo. Herrmann, (Ed.), R.D. Mindlin and Applied Mechanics, Pergamon, New York, 1974, pp. 105 ff.

$5 \mathrm{O}$. Reynolds, On the dilatancy of media composed of rigid particles in contact, Philos. Mag., Ser. 5, 20 (1885) 469-481.

6 R.D. Mindlin, Compliance of elastic bodies in contact, J. Appl. Mech. (Trans. ASME), 16 (1949) 259-268.

7 R.D. Mindlin, Mechanics of granular media, Proc. Second U.S. National Congress of Appl. Mech., 1954, pp. 13-20.

8 M.D.A. Cooley and M.E. O'Neill, On the slow rotation of a sphere about a diameter parallel to a nearby plane wall, J. Inst. Math. Its Appl., 4 (1968) 163-173.

9 M.E. O'Neill and K. Stewartson, On the slow motion of a sphere parallel to a nearby plane wall, J. Fluid Mech., 27 (1967) 705-724.

$10 \mathrm{H}$. Christensen, Elastohydrohynamic theory of spherical bodies in normal approach, J. Lubr. Technol. (Trans. ASME, Ser. F), 92 (1970) 145-154.

11 J.D. Goddard and C. Miller, Non-linear effects in the rheology of dilute suspensions, J. Fluid Mech., 28 (1967) 657-763.

12 S.P. Timoshenko and J.N. Goodier, Theory of Elasticity, 3rd edn., McGraw-Hill, New York, 1970, pp. $398 \mathrm{ff}$.

13 A.E.H. Love, A Treatise on the Mathematical Theory of Elasticity, 4th edn., Dover, New York, 1927.

14 J.L. Lubkin, Contact Problems. In W. Flugge (Ed.), Handbook of Eng. Mech., McGrawHill, New York, 1962, Chap. 42.

15 J. Boussinesq, Applications des Potentiels à l'Etude de l'Equilibre et du Movement des Solides Elastiques, Gauthier-Villars, Paris, 1885.

16 J.C. Bell and J.W. Kannel, Simulation of ball bearing lubrication with a rolling disk apparatus, J. Lubr. Technol. (Trans. ASME, Ser. F), 92 (1970) 1--15.

17 H. Brenner and D.W. Condiff, Transport mechanics in systems of orientable particles. IV. Convective transport, J. Colloid Interface Sci., 47 (1974) 199-264.

18 P.L. Kapitza, Hydrodynamic theory of lubrication during rolling, $\mathrm{Zh}$. Tekh. Fiz., 25 (1955) 747-762.

19 H. Deresiewicz, Mechanics of Granular Matter, Advan. Appl. Mech., Vol. 5, Academic Press, New York, 1958, pp. 233-306.

20 H. Deresiewicz, On the mechanics of granular media, Appl. Mech. Surv., (1966) 277-284. 